\title{
Hyperbilirubinemia in atazanavir treated HIV-infected patients: the impact of the UGTIAI*28 allele
}

This article was published in the following Dove Press journal:

Pharmacogenomics and Personalized Medicine

20 June 2017

Number of times this article has been viewed

\section{Periklis Panagopoulos' \\ Efstathios Maltezos' \\ Angelos Hatzakis ${ }^{2}$ \\ Dimitrios Paraskevis ${ }^{2}$}

' $2^{\text {nd }}$ Department of Internal Medicine, Democritus University of Thrace,

Alexandroupoli, Greece; ${ }^{2}$ Department of Hygiene, Epidemiology and Medical Statistics, Medical School, National and Kapodistrian University of Athens, Athens, Greece
Correspondence: Dimitrios Paraskevis Department of Hygiene, Epidemiology and Medical Statistics, Medical School, National and Kapodistrian University of Athens, 75 Mikras Asias Street, GR-I I527, Athens, Greece Phone +302107462119 Email dparask@med.uoa.gr

\begin{abstract}
Combination antiretroviral treatment (cART) has significantly improved the life expectancy of people living with HIV. The life-long nature of cART increases the risk of side effects, which in some cases may have been caused by specific genetic characteristics. Patients treated with atazanavir (ATV) boosted with ritonavir (rit), which is a protease inhibitor used for the treatment of HIV, present with elevated bilirubin levels, at high proportions. ATV/ritrelated hyperbilirubinemia has been previously associated with genetic characteristics in uridine diphosphate glucuronosyltransferase (UGT) enzyme. The prevalence of the UGT1A1*28 variant, which is the most frequent polymorphism in the UGT1A1 superfamily, has been found to range between $9 \%$ and $\sim 60 \%$ with the highest frequency in Africa. Pharmacokinetics for additional HIV drugs, such as the integrase inhibitors Raltegravir and Elvitegravir, has been also shown to be influenced by UGT1A1 polymorphisms. Pharmacogenetics/pharmacogenomics testing can be useful to identify a patient's susceptibility to drug toxicity and therefore to facilitate selection of the optimal long-term suppressive regimen.
\end{abstract}

Keywords: atazanavir, hyperbilirubinemia, pharmacogenomics

\section{Introduction}

The introduction of combination antiretroviral therapy (cART) has significantly prolonged the life-expectancy of people living with HIV (PLWHIV). Since the early introduction of cART in the middle 1990s, numerous drugs and drug classes have become available, with improved efficacy and drug characteristics modifying the face of HIV-1 infection to a chronic disease. ${ }^{1}$ The benefits of cART, however, remain only under lifelong administration of treatment. Given the chronic status of treatment, previous studies have investigated the pharmacogenetics of ART namely the genetic characteristics associated with differences in response or side effects of drugs. Some of the earliest reports in 2002, showed that genetic variations in HLA-B have been associated with hypersensitivity to abacavir (ViiV Healthcare, London, UK). ${ }^{2}$

Based on these findings pharmacogenetics or pharmacogenomics have been routinely implemented for the clinical management of HIV infection. Whilst the benefits of ART to prolong the life expectancy of PLWHIV, side effects may cause a need for treatment switch. Given that in some cases side effects may appear due to specific characteristics in human genome, the rationale for treatment switch is based upon modifying or optimizing an antiretroviral regimen according to patient's genetic analysis. Genetic testing can provide useful information about a patient's susceptibility to an adverse event related to a component of the regimen. ${ }^{2}$ This is important, as we 
should provide a life-long term therapy. Pharmacogenetics become of key importance as a step to personalize medicine: the right drug at the right dose for the right patient.

Atazanavir (ATV; Bristol-Myers Squibb Pharmaceuticals, New York, NY, USA) boosted with low dose of ritonavir (rit; Abbvie Biopharmaceutical, Lake Bluff, IL, USA) is a protease inhibitor (PI), which has been recommended for almost a decade as part of first-line/preferred/recommended regimen in drug-naïve PLWHIV. ${ }^{3}$ More than half of patients on ATV experience elevated bilirubin levels, which can reach grade 3-4 in approximately one third of all cases. ${ }^{4}$ In ACTG 5237 study, $8 \%$ of the patients discontinued ATV due to jaundice. ${ }^{5}$ The mechanism of this adverse event resembles Gilbert's Syndrome, a form of mild unconjugated hyperbilirubinemia that affects $\sim 3 \%-9 \%$ of individuals with European ancestry. ${ }^{6}$

\section{Background}

ATV acts as an inhibitor of uridine diphosphate glucuronosyltransferase (UGT), the enzyme responsible for hepatic conjugation of bilirubin. Three UGT subfamilies have been identified based on their genetic similarity: UGT1A, UGT2A, UGT2B. The major UGT1A subfamily enzyme, UGT1A1, is expressed mainly in the liver and gastrointestinal track and is efficient for the effective elimination of bilirubin. ${ }^{7}$ The most frequent genetic variant that affects UGT1A1 function is a dinucleotide $\mathrm{TA}_{\mathrm{n}}$ repeat polymorphism (rs8175347), which varies from 5 to 7 TA repeats. The TA repeats are located 53 nucleotides upstream the translation start. The wild-type allele $\left(U G T 1 A 1^{*} 1\right)$ has six (TA) repeats. The $\mathrm{TA}_{7}\left(U G T 1 A 1^{*} 28\right)$ is one of the frequent polymorphisms, recognized as a causative genetic variant of Gilbert's Syndrome. The $\mathrm{TA}_{5}(\mathrm{UGT1A} 1 * 36)$ and $\mathrm{TA}_{8}(\mathrm{UGT1A} 1 * 37)$ are rare or absent across different populations. Previous studies have shown that the $\mathrm{TA}_{7}(\mathrm{UGT} 1 \mathrm{~A} 1 * 28)$ allele causes lower gene transcription than $\mathrm{TA}_{6}(\mathrm{UGT} 1 \mathrm{~A} 1 * 1)$ allele. ${ }^{8}$ Individuals homozygous to $\left(\mathrm{TA}_{7} / \mathrm{TA}_{7}\right)$ are at higher risk than heterozygous patients $\left(\mathrm{TA}_{6} / \mathrm{TA}_{7}\right) \cdot{ }^{9}$

Several studies have confirmed the association of hyperbilirubinemia with the use of ATV/rit-containing regimens, in individuals carrying the aforementioned polymorphism, although fewer recorded the prevalence of UGT1A1 polymorphism in PLWHIV. The prevalence of the UGTA1A1*28 variant was previously estimated to be $\sim 60 \%$ among HIV-infected individuals in Greece, ${ }^{10}$ which is similar to estimations based on national data for cancer patients' cohorts and healthy controls (58.2\% vs $51.3 \%$ and $62.5 \%$, respectively). ${ }^{11,12}$ The prevalence of UGTA $1 \mathrm{~A} 1 * 28$ allele ranges from $9 \%$ to $56 \%$, with the prevalence to be higher in Africans. Another study reported that the UGT1A1*1 $\mathrm{TA}_{6}$ allele was most prevalent in whites (69\%) versus $47 \%$ in Africans, and 60\% in Hispanics, followed by UGT1A1*28 $\mathrm{TA}_{7}$ (31\% in whites, $40 \%$ in Africans, and 39\% in Hispanics). ${ }^{13}$ An association between UGTA1A1*28 polymorphism and ATV/rit discontinuation was found only in Hispanics. Furthermore subjects with two copies of the allele variant (UGT1A1 28 homozygotes) had the highest risk versus the $U G T 1 A 1 * 28$ heterozygotes showed intermediate risk for developing hyperbilirubinaemia. ${ }^{14}$ Conversely, the best pre-treatment predictor of peak on-treatment indirect bilirubin $>3.0 \mathrm{mg} / \mathrm{dL}$ was the combination of rs887829 TT homozygosity, greater bilirubin concentration, and greater hemoglobin concentration ${ }^{15}$ (Table 1).

\section{Discussion}

Genome-wide association studies (GWAS) have found that an SNP rs887829 (c-364C>T; UGT1A1*80), which is close to the $\mathrm{TA}_{\mathrm{n}}$ repeats, is associated with indirect hyperbilirubinemia in the general population. The rs887829 $\mathrm{T}$ allele was linked with the $\mathrm{TA}_{7}$ and $\mathrm{TA}_{8}$ alleles, while rs887829 $\mathrm{C}$ was linked with the $\mathrm{TA}_{5}$ and $\mathrm{TA}_{6}$ alleles. ${ }^{16-18}$ Additional polymorphisms have been described to be associated with increased bilirubin

Table I Major studies describing the impact of UGTIAI*28 allele

\begin{tabular}{|c|c|c|}
\hline Author & Title & Citation \\
\hline Hsieh et $\mathrm{al}^{8}$ & $\begin{array}{l}\text { Molecular pathogenesis of Gilbert's syndrome: decreased TATA-binding } \\
\text { protein binding affinity of UGTIAI gene promoter }\end{array}$ & Pharmacogenet Genomics. 2007;17(4):229-236 \\
\hline Sanna et al $^{18}$ & $\begin{array}{l}\text { Common variants in the SLCOIB3 locus are associated with bilirubin levels } \\
\text { and unconjugated hyperbilirubinemia }\end{array}$ & Hum Mol Genet. 2009; I8(|4):27II-27I8 \\
\hline Kang et $\mathrm{al}^{19}$ & Genome-wide association of serum bilirubin levels in Korean population & Hum Mol Genet. 20I0;19(I8):3672-3678 \\
\hline Chen et $\mathrm{al}^{17}$ & UGTIAI is a major locus influencing bilirubin levels in African Americans & Eur J Hum Genet. 20I2;20(4):463-468 \\
\hline Dai et $\mathrm{al}^{20}$ & $\begin{array}{l}\text { A genome-wide association study for serum bilirubin levels and gene- } \\
\text { environment interaction in a Chinese population }\end{array}$ & Genet Epidemiol. 2013;37(3):293-300 \\
\hline Panagopoulos et al ${ }^{10}$ & $\begin{array}{l}\text { High prevalence of the UGTIAI*28 variant in HIV-infected individuals in } \\
\text { Greece }\end{array}$ & Int J STD AIDS. 20|4;25(I2):860-865 \\
\hline Kanestri et al $^{9}$ & $\begin{array}{l}\text { Clinical significance of the UGTIAI*28 allele detection in HIV-infected } \\
\text { patients }\end{array}$ & J Int AIDS Soc. 2014;17(4 Suppl 3):19579 \\
\hline
\end{tabular}


concentrations. Specifically, rs11891311 and rs6742078 were found in a Korean and a Chinese population, respectively, both of which were linked with rs887829; polymorphism rs4148323 (c.211G>A; p.Gly71Arg; UGT1A1*6) was also found to be an independent predictor of bilirubin concentrations in Asian populations (Korea and China). ${ }^{19,20}$ The cornerstone studies regarding the impact of the allele polymorphisms are included in Table 1.

Discontinuation rates of $\mathrm{ATV} / \mathrm{rit}$ ranged from $<1 \%$ to $9 \%$. On the contrary switching to unboosted ATV reduces bilirubin without compromising treatment efficacy. ${ }^{21} \mathrm{~A}$ recent study in British Columbia showed that this switch may have favorable effects on bilirubin and renal function. ${ }^{22}$ Notably, another option is to switch from a standard dose of ATV (300 mg) to a low dose of ATV (200 mg) boosted with a low dose of rit (100 mg) with similar results. ${ }^{23}$ Additionally chronic oxidative stress (OS) may play a role in cardiovascular disease in HIV-infected patients and increased bilirubin levels may have a beneficial role in counteracting OS. ${ }^{24}$ Changes in bilirubin and changes in OS markers (lipoproteinassociated phospholipase 2, myeloperoxidase and oxidized low-density lipoprotein) were significantly correlated.

ATV-associated indirect hyperbilirubinemia does not indicate hepatic injury, but some patients are not prescribed ATV to avoid the possibility of jaundice. Implications of UGT1A1 genetic testing for prescribing ATV, boosted with rit or cobicistat (Gilead Sciences, Foster City, CA, USA) may be influenced by several factors such as consequences of jaundice for a particular patient receiving therapy with additional PIs (eg, darunavir [Janssen Pharmaceutical, Beerse, Belgium]) or integrase inhibitors. However, in this population bilirubin could represent an adherence biomarker. For example, PLWHIV on with ATV failure of plasma bilirubin to increase from baseline (regardless of UGT1A1 genotype) is strong evidence that ATV/rit or ATV/cobicistat was not taken during the prior $\sim 24$ hours. ${ }^{25}$

The cost-effectiveness of a priori pharmacogenetic testing for UGT1A1 polymorphism was assessed by Schackman et al. ${ }^{26}$ They concluded that such an approach could be cost-effective only if the assay cost is low and if it contributes to better in-treatment retention rates, provided other comparator choices have the same drug cost and efficacy. Regarding the characteristics of genetic testing, previous studies suggested that the pooled predictive value of two UGT1A1*28 alleles for severe ATV-associated unconjugated hyper bilirubinemia was $40.3 \%$ and the pooled negative predictive value was $88.1 \%$. $^{7}$ Most physicians believe that genetic testing can be helpful before the prescription of ATV.
On the other hand, ATV is not the only antiretroviral influenced by UGT polymorphisms. The effect of demographic and clinical covariates, as well as frequent genetic polymorphisms in six genes (CYP3A4*22, CYP3A5*3, CYP2C19*2, CYP2C19*17, UGT1A1*28, UGT1A4*2) has been investigated on rilpivirine (Janssen Pharmaceutical) elimination; the results showed low variability in its pharmacokinetics. ${ }^{26}$ Elvitegravir (Gilead Sciences), an HIV integrase inhibitor, is also metabolized primarily by CYP3A and secondarily by UGT1A1/3. ${ }^{27}$ Raltegravir (RAL; Merck \& Co, Kenilworth, NJ, USA), another HIV integrase inhibitor, is metabolized mainly by UGT1A1. A study in Japanese HIV-infected patients showed that the presence of one or two alleles of UGT1A1*6 were independent factors associated with high RAL plasma trough concentrations $(>0.17 \mu \mathrm{g} / \mathrm{mL})$. Of note in Japanese population the percentage of homozygotes for UGT1A $1 * 6$ and UGT1A $1 * 28$ was $6 \%$ and $4 \%$, respectively, and that of heterozygotes was $22 \%$ and $17 \%$, respectively. ${ }^{28}$

\section{Conclusion}

Pharmacogenomics and genetics are useful not only for the assessment of the patient's individual response to a specific drug combination but also in their ability to identify individual susceptibility to drug toxicity. UGT1A1 polymorphisms testing should be performed before the initial administration of an antiretroviral regimen in PLWHIV as recommended by HIV treatment guidelines.

\section{Disclosure}

The authors report no conflicts of interest in this work.

\section{References}

1. Deeks SG, Lewin SR, Havlir DV. The end of AIDS: HIV infection as a chronic disease. Lancet. 2013;382(9903):1525-1533.

2. Hetherington S, Hughes AR, Mosteller M, et al. Genetic variations in HLA-B region and hypersensitivity reactions to abacavir. Lancet. 2002;359(9312):1121-1122.

3. EACS. European AIDS Clinical Society Guidelines for the treatment of HIV infected adults in Europe.Version 8.2 [webpage on the Internet]. Available from http://www.eacsociety.org/guidelines/eacs-guidelines/ eacs-guidelines.html (last update 4/10/2016). Accessed May 30, 2017.

4. Soriano V, Arasteh K, Migrone H, et al. Nevirapine versus atazanavir/ ritonavir, each combined with tenofovir disoproxil fumarate/emtricitabine, in antiretroviral-naive HIV-1 patients: the ARTEN Trial. Antivir Ther. 2011;16(3):339-348.

5. Lennox JL, Landovitz RJ, Ribaudo HJ, et al. Efficacy and tolerability of 3 nonnucleoside reverse transcriptase inhibitor-sparing antiretroviral regimens for treatment-naive volunteers infected with HIV-1: a randomized, controlled equivalence trial. Ann Intern Med. 2014;161(7): $461-471$.

6. Bosma PJ, Chowdhury JR, Bakker C, et al. The genetic basis of the reduced expression of bilirubin UDP-glucuronosyltransferase 1 in Gilbert's syndrome. N Engl J Med. 1995;333(18):1171-1175. 
7. Gammal RS, Court MH, Haidar CE, et al. Clinical Pharmacogenetics Implementation Consortium (CPIC) Guideline for UGT1A1 and Atazanavir Prescribing. Clin Pharmacol Ther. 2016;99(4):363-369.

8. Hsieh TY, Shiu TY, Huang SM, et al. Molecular pathogenesis of Gilbert's syndrome: decreased TATA-binding protein binding affinity of UGT1A1 gene promoter. Pharmacogenet Genomics. 2007;17(4):229-236.

9. Kanestri V, Mironov K, Kravchenko A, et al. Clinical significance of the UGT1A $1 * 28$ allele detection in HIV-infected patients. J Int AIDS Soc. 2014;17(4 Suppl 3):19579.

10. Panagopoulos P, Paraskevis D, Katsarolis I, et al. High prevalence of the UGT1A $1 * 28$ variant in HIV-infected individuals in Greece. Int $J$ STD AIDS. 2014;25(12):860-865.

11. Karatzas A, Giannatou E, Tzortzis V, et al. Genetic polymorphisms in the UDP-glucuronosyltransferase 1A1 (UGT1A1) gene and prostate cancer risk in Caucasian men. Cancer Epidemiol. 2010;34(3):345-349.

12. Tsezou A, Tzetis M, Giannatou E, et al. Gilbert syndrome as a predisposing factor for cholelithiasis risk in the Greek adult population. Genet Test Mol Biomarkers. 2009;13(1):143-146.

13. Ribaudo HJ, Daar ES, Tierney C, et al. Impact of UGT1A1 Gilbert variant on discontinuation of ritonavir-boosted atazanavir in AIDS Clinical Trials Group Study A5202. J Infect Dis. 2013;207(3):420-425.

14. Busti AJ, Hall RG, Margolis DM. Atazanavir for the treatment of human immunodeficiency virus infection. Pharmacotherapy. 2004;24(12):1732-1747.

15. Johnson DH, Venuto C, Ritchie MD, et al. Genomewide association study of atazanavir pharmacokinetics and hyperbilirubinemia in AIDS Clinical Trials Group protocol A5202. Pharmacogenet Genomics. 2014;24(4):195-203.

16. Johnson AD, Kavousi M, Smith AV, et al. Genome-wide association metaanalysis for total serum bilirubin levels. Hum Mol Genet. 2009;18(14): 2700-2710.

17. Chen G, Ramos E, Adeyemo A, et al. UGT1A1 is a major locus influencing bilirubin levels in African Americans. Eur J Hum Genet. 2012;20(4):463-468.

18. Sanna S, Busonero F, Maschio A, et al. Common variants in the SLCO1B3 locus are associated with bilirubin levels and unconjugated hyperbilirubinemia. Hum Mol Genet. 2009;18(14):2711-2718.
19. Kang TW, Kim HJ, Ju H, et al. Genome-wide association of serum bilirubin levels in Korean population. Hum Mol Genet. 2010;19(18): 3672-3678.

20. Dai X, Wu C, He Y, et al. A genome-wide association study for serum bilirubin levels and gene-environment interaction in a Chinese population. Genet Epidemiol. 2013;37(3):293-300.

21. Ferraris L, Vigano O, Peri A, et al. Switching to unboosted atazanavir reduces bilirubin and triglycerides without compromising treatment efficacy in UGT1A $1 * 28$ polymorphism carriers. J Antimicrob Chemother. 2012;67(9):2236-2242.

22. Harris M, Ganase B, Watson B, et al. Efficacy and safety of "unboosting" atazanavir in a randomized controlled trial among HIV-infected patients receiving tenofovir DF. HIV Clin Trials. 2017;18(1):39-47.

23. Bunupuradah T, Kiertiburanakul S, Avihingsanon A, et al. Low-dose versus standard-dose ritonavir-boosted atazanavir in virologically suppressed Thai adults with HIV (LASA): a randomised, open-label, non-inferiority trial. Lancet HIV. 2016;3(8):e343-350.

24. Estrada V, Monge S, Gomez-Garre MD, et al. Relationship between plasma bilirubin level and oxidative stress markers in HIV-infected patients on atazanavir- vs. efavirenz-based antiretroviral therapy. $H I V$ Med. 2016;17(9):653-661.

25. Morello J, Alvarez E, Cuenca L, et al. Short communication: use of serum bilirubin levels as surrogate marker of early virological response to atazanavir-based antiretroviral therapy. AIDS Res Hum Retroviruses. 2011;27(10):1043-1045.

26. Schackman BR, Haas DW, Becker JE, et al. Cost-effectiveness analysis of UGT1A1 genetic testing to inform antiretroviral prescribing in HIV disease. Antivir Ther. 2013;18(3):399-408.

27. Ramanathan S, Custodio JM, Wei X et al. Pharmacokinetics of coformulted Elvitegravir/Cobocistat/Emtricitabine/Tenofovir Disoproxil Fumarate in healthy subjects. JAcquir Immune Defic Syndr. 2016;72(3): 281-288.

28. Yagura H, Watanabe D, Ashida M et al. Correlation between UGT1A1 polymorphisms and raltegravir plasma trough concentrations in Japanese HIV-1-infected patients. J Infect Chemother. 2015;21(10): 713-717.
Pharmacogenomics and Personalized Medicine

\section{Publish your work in this journal}

Pharmacogenomics and Personalized Medicine is an international, peerreviewed, open access journal characterizing the influence of genotype on pharmacology leading to the development of personalized treatment programs and individualized drug selection for improved safety, efficacy and sustainability. This journal is indexed on the American Chemical

\section{Dovepress}

Society's Chemical Abstracts Service (CAS). The manuscript management system is completely online and includes a very quick and fair peer-review system, which is all easy to use. Visit http://www.dovepress. $\mathrm{com} /$ testimonials.php to read real quotes from published authors. 